\title{
Wellbeing, mental health, and video games: a shifting narrative from player to industry perspective
}

Video games saved 2020 by keeping us sane and connected and further transforming our lives. At the same time, however, the existing flaws within the industry and the underlying digital infrastructure became evermore visible.

In the short history of their existence, video games have consistently been blamed for causing various disorders or antisocial behaviour. This ranges from the early discourse on video games as a cause of violent behaviour, which, while repeatedly debunked by almost 30 years of research, keeps reappearing, to more recent accounts of toxicity and harassment in video game culture and concerns around excessive gameplay. This is illustrated by, for instance, the inclusion by the World Health Organisation of the 'Gaming Disorder' in the 11th Revision of the International Classification of Diseases or China heavily regulating over the past few years the quantity and quality of video games children and young people have access to, quoting the negative impact on physical and mental health of excessive gameplay. Over the course of the last year, however, we have seen a shift in the narrative, from a single-minded focus on the harms allegedly caused by video games to a more granular and balanced approach.

While the industry has been heavily affected by the impact of the global pandemic - from lockdowns to working from home to disruptions to software and hardware releases - it has also provided an opportunity for growth as people turned to video games to pass the time, stay in touch with friends and family, and to expand their world in Cyberspace, given the limited opportunities for interaction and exploration in the physical world.

The conversation about wellbeing, mental health, and video games now includes evidence-based research about the numerous benefits of playing games, ${ }^{1}$ public debate on reducing 'online harms' and an increasing number of video game companies implementing an informed and proactive approach with regards to player and community support. Several initiatives focusing on mental health in video games took place in October this year, running a range of activities and promoting messages on social media to encourage players to find time to play games and talk with their friends to support their mental health.

Despite this welcome shift towards the positive impact of video games, there remains a significant gap in the current narrative: the wellbeing and the mental health of those working in the industry. While industry has experienced an unprecedented growth, the cost of this has been borne by individual developers, who have found themselves working significantly longer hours. According to Renee Gittins, executive director of the International Game Developer Association (IGDA): 'While the game industry has overall done well during the COVID-19 pandemic, we saw nearly double the number of developers reporting they had crunched recently. This sudden loss of progress compared to the past trend of crunch decreasing year over year is likely caused by inefficiencies and delays caused by work from home and mental health challenges caused by the pandemic.' Crunch refers to a form of unpaid overtime where staff in a video game studio are forced to work long hours in order to complete a project on time. While the industry had been making some headway in tackling the problem over the last few years, things appear to have regressed during the pandemic, as Gittins points out. In addition to serious concerns about workload, horrific stories of sexual harassment and discrimination have also emerged from some of the most high-profile studios in the industry. Taken together, these paint a most distressing picture of life as a professional video game developer.

To date, however, these issues have yet to make an impression on the popular imagination. It is telling that the recent blockbuster movie revolving around a video game universe - Free Guy - puts the non-player character (NPC) at the core of the story. The creators, developers and other actors behind the scenes, the industry's work culture and stereotypes serve mainly as a stage set for the bubblegum flavoured quest for freedom and romance.

As we read about Facebook's plans to build a 'metaverse' and hire 10,000 new employees needed for this project, we may consider the trajectory of the interactive 
entertainment industry and its exponential growth. The Big Tech companies are not concerned so much about governments and their laws, regulations, and enforcement agencies, as they are worried about collective action. There is nothing like a walkout or a strike to focus minds in Silicon Valley. Unionized game developers are a small but growing part of the industry and, hopefully, a force to reckon with in the near future.

While we look forward to those developments, in this issue of the Interactive Entertainment Law Review, we bring together a selection of articles that take a close look at different legal, ethical, and regulatory dimensions of video games. Emmanuelle Sarlangue explores 'Registered Community designs in the video game industry: a neglected yet potent tool'. She argues that design rights have not yet been adopted by the industry within their IP strategy and suggests that some legislative changes could alleviate current uncertainties. Olivia Jean-Baptiste's article titled 'Augmented and virtual reality art: a new frontier of legal protection' looks at the current status of protection of art works in virtual and augmented realities and traces the level of moral rights protection of such works in the UK, France and the US. The article by Zoi Potolia 'Deceptive advertising and its connection to unregulated gambling in the gaming industry' argues that influencer advertising has gone too far in relation to promoting gambling and analyses the growing body of cases litigated in the US. Finally, 'Protecting video games against online copyright infringement: a Russian approach to site blocking' by Alena Kuzmina looks at site blocking practices in Russia applied to curb video game piracy.

We hope you enjoy this issue.

Dr Gaetano Dimita Professor Jon Festinger Q.C. Dr Yin Harn Lee Dr Michaela MacDonald Dr Marc Mimler 